\title{
Gemcitabine-Induced Radiation Recall Myositis: Case Report and Review of the Literature
}

\author{
Adarsh Ravishankar ${ }^{a}$ Sean S. Park ${ }^{b} \quad$ Kenneth R. Olivier ${ }^{b}$ \\ Kimberly S. Corbin ${ }^{b}$ \\ aUniversity of Minnesota Medical School, Minneapolis, MN, USA; ${ }^{b}$ Division of Radiation \\ Oncology, Mayo Clinic, Rochester, MN, USA
}

\section{Keywords}

Breast cancer $\cdot$ Gemcitabine $\cdot$ Radiation $\cdot$ Bone metastasis

\begin{abstract}
Gemcitabine-induced radiation recall (GIRR) is a phenomenon wherein the administration of gemcitabine induces an inflammatory reaction within an area of prior radiation. We present the case of a 39-year-old female patient with metastatic breast cancer who experienced GIRR myositis 3 months following postoperative radiotherapy, with additional potential paraspinal myositis following ablative radiotherapy to the thoracic spine. A review of previously published cases of GIRR myositis was performed. The case and literature review describe the clinical course and presentation of GIRR, and highlight the importance of including radiation recall as part of a differential diagnosis when a patient undergoing chemotherapy experiences an inflammatory reaction at a prior site of radiation.

(C) 2018 The Author(s)

Published by S. Karger AG, Basel
\end{abstract}




\section{Case Reports in Oncology}

\section{Introduction}

Radiation recall describes a well-known, but poorly understood phenomenon by which administration of chemotherapy or another systemic agent induces an inflammatory reaction within a previously irradiated field [1]. Radiation recall was first described in 1959, when it was found that latent effects of radiation, such as dermatitis, could be induced by actinomycin D [2]. Though clinically rare, since that time radiation recall has been observed for various other systemic agents and inciting factors [3]. While the recall phenomenon is most commonly reported as dermatitis, it has also been observed in other organs including the lung, intestine, and muscles [1, 4-6]. Gemcitabine has previously been implicated in radiation recall. When reported, it is noted to preferentially affect internal tissue and organs [7]. Herein we report a case of a 39-year-old woman with myositis that is clinically consistent with gemcitabine-induced radiation recall (GIRR) myositis. We also review reported cases of GIRR myositis and discuss the findings and implications.

\section{Case Description}

Events are outlined in the timeline (Fig. 1) and summarized below. In 2008, the 39-yearold patient was diagnosed with metastatic ER-negative, PR-negative, HER2-positive cancer. At initial presentation, PET scan demonstrated both regional lymph node involvement and osseous metastases. She was started on systemic therapy with Adriamycin and Cytoxan, followed by Taxol and Herceptin. Subsequent therapies included maintenance single-agent Herceptin, followed by Herceptin and lapatinib. By late 2011, her osseous disease showed complete radiologic response on PET scan, and she was offered local therapy to the primary tumor. In January 2012, she underwent a right mastectomy, with ypT2N1a disease, followed by postmastectomy radiation up to 50 Gy with a 10 -Gy chest wall boost.

In late August 2012, she presented with a painful osseous lesion at the T12 vertebra and an impending pathologic fracture of the left distal femur. She underwent surgical stabilization of the femur with rod placement, followed by postoperative radiotherapy, 30 Gy in 10 fractions (Fig. 2a). T12 was treated with single-fraction stereotactic body radiotherapy (SBRT) up to 24 Gy.

In December 2012, gemcitabine $\left(1,000 \mathrm{mg} / \mathrm{m}^{2}\right.$, days 1 and 8$)$ and Herceptin were given in a 21-day cycle. In January 2013, she presented to the Emergency Department with leg swelling, shortness of breath, and pleuritic chest pain to the lower thorax. Physical examination noted left leg swelling and some mild effusion of the left knee. The ultrasound was negative for deep vein thrombosis (DVT), and the chest CT was negative for pulmonary embolism. Symptoms were attributed to a viral syndrome with associated pleurisy.

The patient's symptoms had worsened at the time of her reexamination by oncology on March 2013. PET scan showed increased FDG activity and edema in the left thigh that was thought to be consistent with postradiation inflammatory changes (Fig. 2b). MRI of the thoracic spine demonstrated paraspinal muscle fluid collections and a new T12 fracture, and chemotherapy was held. CT-guided biopsy of the paraspinal fluid collection was negative for abscess or malignancy. Although a T12 vertebroplasty improved the chest/back pain and her swelling, her leg pain persisted. 


\section{Case Reports in Oncology}

Case Rep Oncol 2018;11:168-178

DOI: $10.1159 / 000487478$

(c)

2018 The Author(s). Published by S. Karger AG, Basel www.karger.com/cro

Ravishankar et al.: Gemcitabine-Induced Radiation Recall Myositis: Case Report and

Review of the Literature

On March 19, 2013, gemcitabine and Herceptin were resumed with a 20\% gemcitabine dose reduction. Within 2 days, she developed worsening leg pain and swelling, and required the use of a cane for ambulation. Repeat extremity ultrasound showed no evidence of DVT, but revealed marked, diffuse enlargement of the vastus medialis or rectus femoris muscles of the left thigh, suggestive of myositis. At that time, her providers noted that her symptoms appeared and worsened following each gemcitabine administration, but improved off chemotherapy. Given the temporal relationship and other negative evaluation, the patient's symptoms were attributed to GIRR from the prior palliative radiotherapy to the left femur. Gemcitabine was stopped, and she was started on dexamethasone and physical therapy. The patient required dexamethasone for relief of her symptoms until August 2013. After a slow taper, she regained leg function and was not rechallenged with gemcitabine.

Over the subsequent 3 years, she had received treatment with Herceptin, ado-trastuzumab, and eribulin/Herceptin without recurrence of the symptoms. Gait was described as normal at the most recent follow-up, and she was described as physically active, engaging in regular exercise. In retrospect, it is also possible that the exacerbation of pain and fluid collection within the paraspinal muscles of T12 seen in March of 2013 may have been related to recall myositis in that region.

\section{Literature Review}

A literature search was performed using the search terms "gemcitabine," "radiation," "recall," and "myositis" to compile known reports of GIRR myositis (Table 1, Table 2, Table 3). Twenty-one cases were identified as having myositis in a radiation port following gemcitabine treatment. Fractionated radiation doses ranged from 28 to $70.2 \mathrm{~Gy}$.

The time interval between radiation and chemotherapy initiation ranged from 0 days to 4 months. The majority had chemotherapy more than 1 month after radiotherapy. The time between radiation and onset of recall symptoms varied from 4 weeks to 5.5 months. Only 1 patient had symptoms less than 1 month after radiation, 20 were $\geq 3$ months, 10 were $\geq 4$ months, and 7 were $\geq 5$ months. All patients achieved at least partial improvement of their symptoms with discontinuation of gemcitabine, regardless of whether their treatment consisted of steroids ( 9 patients), NSAIDs ( 5 patients), analgesics ( 4 patients), or no treatment at all ( 2 patients). Out of the 9 confirmed patients who resumed chemotherapy following myositis, 5 patients underwent gemcitabine rechallenge, with only 1 patient requiring concomitant steroids for symptom control.

The patient described in the present report is consistent with other reported cases. The time intervals between chemotherapy, radiotherapy, and the onset of symptoms are within the range of the other cases. The current case of possible myositis at T12 was the only case associated with a single fraction of ablative radiotherapy. Interestingly, our patient had prior chest wall radiotherapy, but did not develop evidence of recall in that location. In review of the literature, this is consistent with other cases in which recall myositis did not appear in all prior sites of radiotherapy. Remote courses were less often affected by recall myositis. These findings are also consistent with prior reviews of radiation recall that suggest a possible relationship between the time interval from radiation to chemotherapy and the severity of recall symptoms $[1,3,8]$. However, such a correlation likely depends on the rela- 


\section{Case Reports in Oncology}

tionship between several factors, such as drug type, dosage, radiation location, radiation dosage, and timing of each treatment [3].

\section{Discussion}

Radiation recall is a delayed inflammatory response at a site of prior radiation [9, 10]. GIRR has been noted to have a predilection for soft tissue and internal organs, resulting in a less typical appearance of radiation recall. For example, GIRR has been documented to cause pseudocellulitis, acute ascending colitis, abdominal wall and subcutaneous fat stranding, optic neuritis, lymphangitis, rectal hemorrhage, brainstem radionecrosis, and myositis $[5,9,11-13]$. These atypical presentations highlight the importance of clinical awareness of GIRR.

The diagnosis of GIRR myositis is challenging, as it may appear similar to infection, thrombosis, or other sources of inflammation [14]. A few key signs may help distinguish this condition. Primarily, the inflammation is confined to a prior radiation portal. Furthermore, there is a temporal relationship between the timing of gemcitabine administration and appearance of symptoms [9]. Radiologic studies may be used to support the diagnosis of myositis with appearance of swelling and edema of the underlying musculature. They may also assist in ruling out other potential etiologies [15]. Because reported cases of myositis have been associated with elevated creatinine kinase and even compartment syndrome, appropriate recognition and treatment is essential to facilitate recovery and minimize morbidity $[10,16,17]$. Review of cases suggests that the most important factor for resolution is the discontinuation of gemcitabine, with the potential use of anti-inflammatory medications.

The mechanisms of action of radiation recall remain poorly understood. However, the varied presentations of GIRR, even within an individual's prior sites of radiotherapy, suggest that multiple factors influence both the appearance and severity of recall. Jeter et al. [11] proposed that the doses of gemcitabine of $600 \mathrm{mg} / \mathrm{m}^{2}$ or higher may pose a higher recall risk. Others suggest that higher radiation treatment doses or shorter intervals between radiotherapy and chemotherapy may influence recall development [9]. However, these are not well-defined risk factors. With an increased use of gemcitabine across many tumor types, along with routine use of definitive and palliative radiotherapy, clinicians should be alerted to this possible complication.

We suspect that the exacerbation of pain at the site of T12 SBRT along with the MRI and biopsy findings are consistent with a focal recall myositis at that location. Our patient did not have further exacerbation of pain at that site with the dose-reduced gemcitabine. This suggests that the intervention with vertebroplasty may have affected the local environment. The underlying recall mechanism may also have differed from that in the leg. Radiation recall at an SBRT-treated site had been reported in the past, although the recall effect was induced by sorafenib and manifested itself in the form of dermatitis [18].

Due to its rare and unpredictable onset, radiation recall continues to be a poorly understood phenomenon. This is further complicated by the possibility that there are multiple mechanisms for different medications (gemcitabine, carboplatin, etc.) and/or types of recall (dermatitis, myositis, etc.). Current hypotheses for explaining radiation recall include sensitivity of descendants of cells that survive radiation, changes in local vasculature, and drug- 
induced hypersensitivity reactions [1]. There is not enough evidence to support any definite mechanism [1,9]. However, the drug-induced hypersensitivity hypothesis appears to best explain the characteristics of radiation recall. This hypothesis describes radiation recall as a nonimmune inflammatory reaction triggered by certain drugs at a site where the inflammatory threshold has been lowered by radiation. Prior radiation to a specific site may induce constant low-level expression of several inflammatory cytokines, including IL-1, IL-6, PGDF$\beta$, TNF- $\alpha$, and TGF- $\beta$ [18]. Introduction of drugs such as gemcitabine may then lead to upregulation of such inflammatory cytokines inducing the recall reaction [1]. This theory is further supported by the various clinical presentations of radiation recall, including the timing of onset, the lack of worsening reactions following rechallenge in some cases, and the induction of recall from noncytotoxic agents (such as simvastatin) $[1,8]$.

\section{Conclusion}

In conclusion, GIRR myositis is a rare, but significant reaction that may present like other conditions such as DVT or infection. As such, it should be part of the differential diagnosis when a patient on gemcitabine reports pain and swelling to a previously irradiated area, although other causes should be thoroughly investigated. In particular, GIRR myositis should be considered when there is a shorter time interval between the completion of radiation and the initiation of chemotherapy. Discontinuation of chemotherapy and possibly the use of anti-inflammatory medications are important steps to reduce symptoms and improve the patient's quality of life. In some cases, patients have been successfully rechallenged with gemcitabine, although that may not always be possible.

\section{Statement of Ethics}

The authors have no ethical conflicts to disclose. IRB approval was obtained.

\section{Disclosure Statement}

The authors declare that there is no conflict of interest. 


\section{References}

1 Azria D, Magné N, Zouhair A, Castadot P, Culine S, Ychou M et al. Radiation recall: a well recognized but neglected phenomenon. Cancer Treat Rev. 2005 Nov;31(7):555-70.

2 D’Angio GJ, Farber S, Maddock CL. Potentiation of x-ray effects by actinomycin D. Radiology. 1959 Aug; 73(2):175-7.

3 Burris HA 3rd, Hurtig J. Radiation recall with anticancer agents. Oncologist. 2010;15(11):1227-37.

4 Kundak I, Oztop I, Soyturk M, Ozcan MA, Yilmaz U, Meydan N et al. Paclitaxel-carboplatin induced radiation recall colitis. Tumori. 2004 Mar-Apr;90(2):256-8.

5 Grover S, Jones JA, Teitelbaum U, Apisarnthanarax S. Radiation recall myositis: two sites, one patient. Pract Radiat Oncol. 2015 Jan-Feb;5(1):39-42.

6 Schweitzer VG, Juillard GJ, Bajada CL, Parker RG. Radiation recall dermatitis and pneumonitis in a patient treated with paclitaxel. Cancer. 1995 Sep;76(6):1069-72.

7 Friedlander PA, Bansal R, Schwartz L, Wagman R, Posner J, Kemeny N. Gemcitabine-related radiation recall preferentially involves internal tissue and organs. Cancer. 2004 May;100(9):1793-9.

8 Camidge R, Price A. Characterizing the phenomenon of radiation recall dermatitis. Radiother Oncol. 2001 Jun;59(3):237-45.

9 Burris HA 3rd, Hurtig J. Radiation recall with anticancer agents. Oncologist. 2010;15(11):1227-37.

10 O’Regan KN, Nishino M, Armand P, Kelly PJ, Hwang DG, Di Salvo D. Sonographic features of pectoralis muscle necrosis secondary to gemcitabine-induced radiation recall: case report and review of current literature. J Ultrasound Med. 2010 Oct;29(10):1499-502.

11 Jeter MD, Jänne PA, Brooks S, Burstein HJ, Wen P, Fuchs CS et al. Gemcitabine-induced radiation recall. Int J Radiat Oncol Biol Phys. 2002 Jun;53(2):394-400.

12 Nishimoto K, Akise Y, Miyazawa M, Kutsuki S, Hashimoto S, Uchida A. A Case of Severe Rectal Hemorrhage Possibly Caused by Radiation Recall after Administration of Gemcitabine. Keio J Med. 2016;65(1):16-20.

13 Tan DH, Bunce PE, Liles WC, Gold WL. Gemcitabine-related “pseudocellulitis": report of 2 cases and review of the literature. Clin Infect Dis. 2007 Sep;45(5):e72-6.

14 Squire S, Chan M, Feller E, Mega A, Gold R. An unusual case of gemcitabine-induced radiation recall. Am J Clin Oncol. 2006 Dec;29(6):636.

15 Delavan JA, Chino JP, Vinson EN. Gemcitabine-induced radiation recall myositis. Skeletal Radiol. 2015 Mar; 44(3):451-5.

16 Graf SW, Limaye VS, Cleland LG. Gemcitabine-induced radiation recall myositis in a patient with dermatomyositis. Int J Rheum Dis. 2014 Jul;17(6):696-7.

17 Eckardt MA, Bean A, Selch MT, Federman N. A child with gemcitabine-induced severe radiation recall myositis resulting in a compartment syndrome. J Pediatr Hematol Oncol. 2013 Mar;35(2):156-61.

18 Hsieh CH, Lin SC, Shueng PW, Kuo DY. Recall radiation dermatitis by sorafenib following stereotactic body radiation therapy. Onco Targets Ther. 2014 Jun;7:1111-4.

19 Alco G et al. Gemcitabine induced radiation recall myositis: report of two cases. Int J Hematol Oncol. 2009;19(4):249-53.

20 Fakih MG. Gemcitabine-induced rectus abdominus radiation recall. JOP. 2006 May;7(3):306-10.

21 Fogarty G, Ball D, Rischin D. Radiation recall reaction following gemcitabine. Lung Cancer. 2001 AugSep;33(2-3):299-302.

22 Ganem G, Solal-Celigny P, Joffroy A, Tassy D, Delpon A, Dupuis O. Radiation myositis: the possible role of gemcitabine. Ann Oncol. 2000 Dec;11(12):1615-6.

23 Horan G, Smith SL, Podd TJ. Gemcitabine-induced radiation necrosis of the pectoralis major muscle. Clin Oncol (R Coll Radiol). 2006 Feb;18(1):85.

24 Lock M, Sinclair K, Welch S, Younus J, Salim M. Radiation recall dermatitis due to gemcitabine does not suggest the need to discontinue chemotherapy. Oncol Lett. 2011 Jan;2(1):85-90.

25 Miura G, Matsumoto T, Tanaka N, Emoto T, Kawamura T, Matsunaga N. Two cases of radiation myositis probably induced by recall phenomenon. Nihon Igaku Hoshasen Gakkai Zasshi. 2003 Sep;63(8):420-2.

26 Patel SC et al. Gemcitabine-induced radiation recall myositis in a patient with relapsed nasopharyngeal carcinoma. Pract Radiat Oncol. 2017 Jan-Feb;7(1):e19-e22.

27 Pinson PJ, Griep C, Sanders WH, Lelie B. Myositis as a "radiation-recall phenomenon" following palliative chemotherapy with carboplatin-gemcitabin for non-small-cell pulmonary carcinoma. Ned Tijdschr Geneeskd. 2006 Aug;150(34):1891-4.

28 Welsh JS, Torre TG, DeWeese TL, O’Reilly S. Radiation myositis. Ann Oncol. 1999 Sep;10(9):1105-8. 


\section{Case Reports in Oncology}

Case Rep Oncol 2018;11:168-178

DOI: $10.1159 / 000487478$

(c)

(C) 2018 The Author(s). Published by S. Karger AG, Basel www.karger.com/cro

Ravishankar et al.: Gemcitabine-Induced Radiation Recall Myositis: Case Report and Review of the Literature

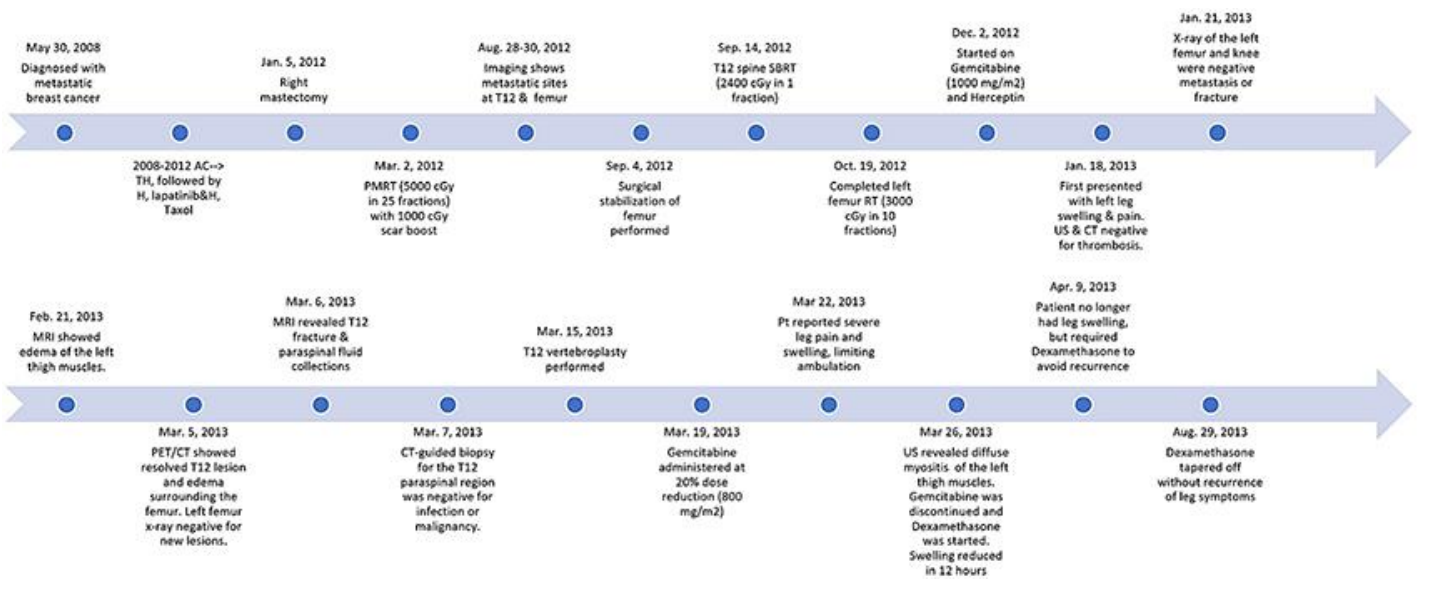

Fig. 1. Timeline of the case. 


\section{Case Reports in Oncology}

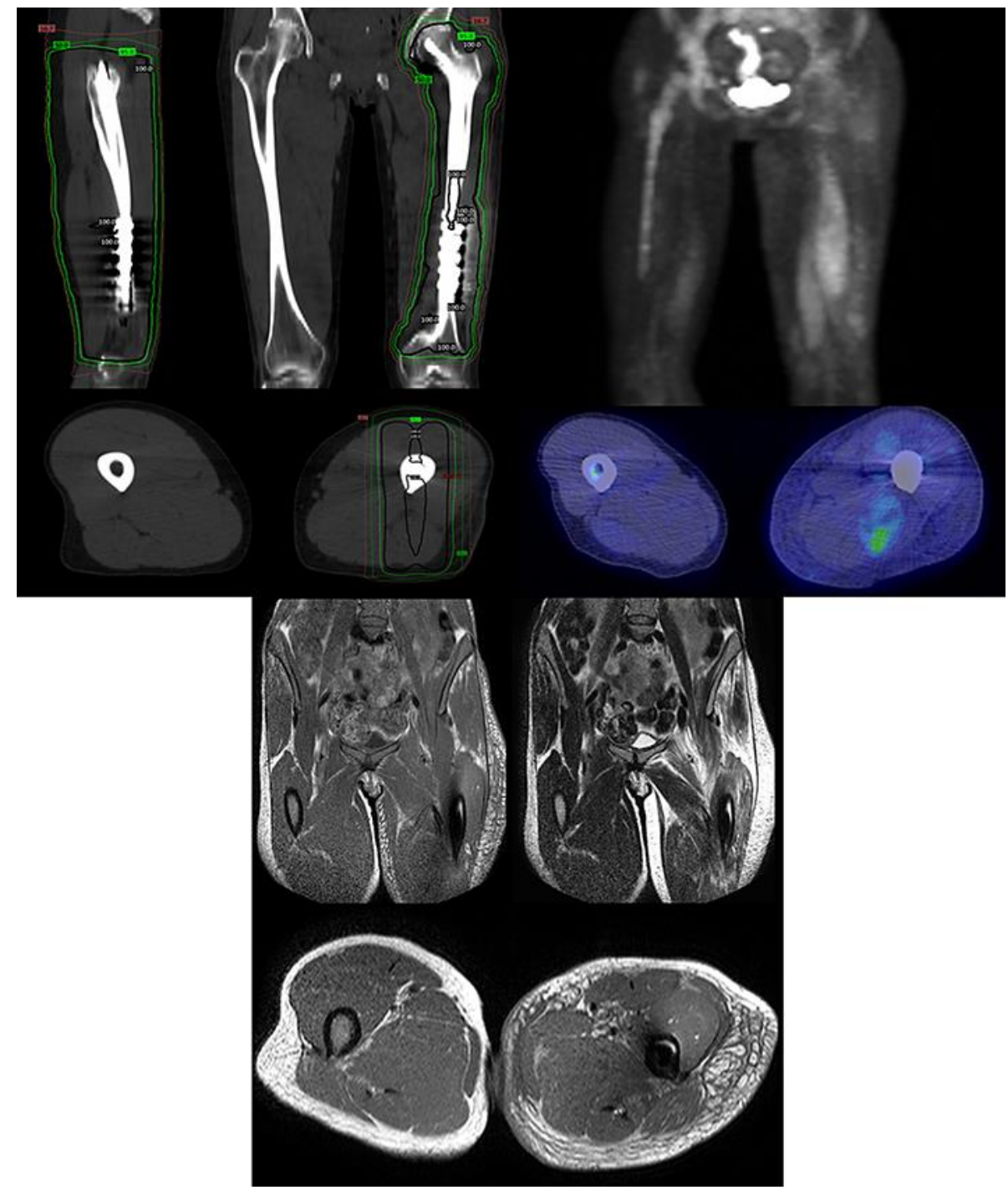

Fig. 2. Top left: radiation fields of the left femur, with sagittal (top left), coronal (top right), and axial (bottom) views. The isodose levels shown are 16.7, 50, 95, and 100\%. Top right: PET/CT showing edema and increased FDG uptake of the left thigh, consistent with myositis, with front-facing 3D (top) and axial (bottom) views. Bottom: MRI showing edema of the left thigh, consistent with myositis, with T1-weighted coronal images (top left), T2-weighted coronal images (top right), and T1-weighted axial images (bottom). 
Ravishankar et al.: Gemcitabine-Induced Radiation Recall Myositis: Case Report and

Review of the Literature

Table 1. Collection of previous case reports of gemcitabine-induced radiation recall myositis

\begin{tabular}{|c|c|c|c|c|c|c|c|c|c|c|c|c|}
\hline Author & $\begin{array}{l}\text { Cancer } \\
\text { type }\end{array}$ & $\begin{array}{l}\text { Radia- } \\
\text { tion } \\
\text { loca- } \\
\text { tion }\end{array}$ & Dose & $\begin{array}{l}\text { CTx } \\
\text { regimen }\end{array}$ & $\begin{array}{l}\text { Time } \\
\text { between } \\
\text { RT and } \\
\text { gemcitabine } \\
\text { start }\end{array}$ & $\begin{array}{l}\text { Time } \\
\text { between } \\
\text { RT com- } \\
\text { pletion } \\
\text { and recall } \\
\text { symptoms }\end{array}$ & $\begin{array}{l}\text { Clinical } \\
\text { symptoms/ } \\
\text { signs }\end{array}$ & $\begin{array}{l}\text { Under- } \\
\text { going chemo } \\
\text { at time of } \\
\text { symptom } \\
\text { onset? }\end{array}$ & $\begin{array}{l}\text { Imaging } \\
\text { findings } \\
\text { (and } \\
\text { modality) }\end{array}$ & $\begin{array}{l}\text { Treat- } \\
\text { ment }\end{array}$ & Outcome & $\begin{array}{l}\text { Post- } \\
\text { treatment } \\
\text { CTx? }\end{array}$ \\
\hline $\begin{array}{l}\text { Alco } \\
\text { et al. } \\
{[19]}\end{array}$ & $\begin{array}{l}\text { Pancre- } \\
\text { atic } \\
\text { adeno } \\
\text { carci- } \\
\text { noma }\end{array}$ & $\begin{array}{l}\text { Pan- } \\
\text { creas } \\
\text { and re- } \\
\text { gional } \\
\text { lymph } \\
\text { nodes }\end{array}$ & $\begin{array}{l}1.8 \mathrm{~Gy} \times \\
25 \\
(45 \mathrm{~Gy})\end{array}$ & $\begin{array}{l}\text { Gemcitabine } \\
1,250 \mathrm{mg} / \mathrm{m}^{2} / \\
\text { week, } 3 \text { weeks } \\
\text { in } 4 \text { week cycle }\end{array}$ & $\begin{array}{l}0 \\
\text { (concurrent) }\end{array}$ & $\begin{array}{l}20 \\
\text { weeks }\end{array}$ & $\begin{array}{l}\text { Tender mass, } \\
\text { pain, and } \\
\text { swelling of } \\
\text { abdominal } \\
\text { muscles }\end{array}$ & $\begin{array}{l}\text { No (last dose } \\
1 \text { month } \\
\text { before } \\
\text { onset) }\end{array}$ & $\begin{array}{l}\text { Edema and } \\
\text { inflammation } \\
\text { of the anterior } \\
\text { and right } \\
\text { abdominal wall } \\
\text { muscles (MRI) }\end{array}$ & $\begin{array}{l}\text { Corticosteroids, } \\
\text { NSAIDs, and } \\
\text { gabapentin }\end{array}$ & $\begin{array}{l}\text { Symptom } \\
\text { reduction in } 1 \\
\text { week; clinical } \\
\text { and radiologic } \\
\text { findings } \\
\text { resolved in } 1 \\
\text { month }\end{array}$ & $\mathrm{N} / \mathrm{A}$ \\
\hline $\begin{array}{l}\text { Alco } \\
\text { et al. } \\
{[19]}\end{array}$ & NSCLC & $\begin{array}{l}\text { Left } \\
\text { upper } \\
\text { lobe } \\
\text { and } \\
\text { ipsilat- } \\
\text { eral L2 } \\
\text { lymph } \\
\text { nodes }\end{array}$ & $\begin{array}{l}62 \mathrm{~Gy} \\
\text { total } \\
\text { (frac- } \\
\text { tions } \\
\mathrm{N} / \mathrm{A} \text { ) }\end{array}$ & $\begin{array}{l}\text { Gemcitabine } \\
\left(1,200 \mathrm{~m} / \mathrm{m}^{2} \text { for }\right. \\
1-8 \text { days), and } \\
\text { carboplatin (AUC } \\
5.5 \text { for days } 1 \text { and } \\
30 \text { ), for } 3 \text { cycles; } \\
\text { gemcitabine } \\
\text { reduced to } 800 \\
\mathrm{mg} / \mathrm{m}^{2} \text { after first cycle } \\
\text { because of intolerance }\end{array}$ & $\begin{array}{l}\text { N/A } \\
\text { (CTx started } \\
\text { after RT) }\end{array}$ & $\begin{array}{l}\text { N/A } \\
(\sim 104 \\
\text { days } \\
\text { after } \\
\text { starting } \\
\text { CTx) }\end{array}$ & $\begin{array}{l}\text { Pain and } \\
\text { swelling of } \\
\text { left breast } \\
\text { and chest wall, } \\
\text { with reduced } \\
\text { ROM of arm } \\
\text { and shoulder }\end{array}$ & $\begin{array}{l}\text { No (last dose } \\
2 \text { weeks } \\
\text { before } \\
\text { onset) }\end{array}$ & $\begin{array}{l}\text { Edema and soft } \\
\text { tissue reaction } \\
\text { at the left } \\
\text { breast } \\
\text { musculature } \\
\text { and subcutane- } \\
\text { ous soft tissue } \\
\text { (MRI) }\end{array}$ & $\begin{array}{l}\text { Corticosteroids, } \\
\text { NSAIDs, } \\
\text { opioids, anti- } \\
\text { histamines, } \\
\text { SOD, } \\
\text { pentoxifylline, } \\
\text { vitamin E, } \\
\text { gabapentin, } \\
\text { topical lidocaine } \\
\text { and selenium }\end{array}$ & $\begin{array}{l}\text { Meds did not } \\
\text { affect myositis; } \\
\text { pain reduced } \\
\text { after } 4 \text { months, } \\
\text { resolved after } 9 \\
\text { months, with } \\
\text { lasting reduced } \\
\text { ROM }\end{array}$ & N/A \\
\hline $\begin{array}{l}\text { Dela- } \\
\text { van } \\
\text { et al. } \\
{[15]}\end{array}$ & $\begin{array}{l}\text { Breast } \\
\text { can- } \\
\text { cer }\end{array}$ & $\begin{array}{l}\text { Left } \\
\text { thigh }\end{array}$ & $\begin{array}{l}8 \mathrm{~Gy} \times 1 \\
(8 \mathrm{~Gy}) \\
(4 \text { years } \\
\text { prior }) \\
3 \mathrm{~Gy} \times 13 \\
(39 \mathrm{~Gy}) \\
(4 \text { months } \\
\text { prior })\end{array}$ & $\begin{array}{l}\text { Gemcitabine } \\
\text { (unknown } \\
\text { dosage) }\end{array}$ & $\begin{array}{l}17 \\
\text { days }\end{array}$ & $\begin{array}{l}107 \\
\text { days }\end{array}$ & $\begin{array}{l}\text { Increasing } \\
\text { pain and } \\
\text { swelling to } \\
\text { the posterior } \\
\text { left thigh, warm } \\
\text { to palpation }\end{array}$ & $\begin{array}{l}\text { No (last dose } \\
\sim 3.5 \text { weeks } \\
\text { before } \\
\text { onset) }\end{array}$ & $\begin{array}{l}\text { Increased } \\
\text { signal } \\
\text { intensity in } \\
\text { the posterior } \\
\text { thigh muscula- } \\
\text { ture (MRI) }\end{array}$ & $\begin{array}{l}\text { Dexameth- } \\
\text { asone }\end{array}$ & $\begin{array}{l}\text { Symptoms } \\
\text { improved } \\
\text { over } 3 \text { days, } \\
\text { symptom free } \\
1 \text { week later }\end{array}$ & $\begin{array}{l}\text { Not re- } \\
\text { ported }\end{array}$ \\
\hline $\begin{array}{l}\text { Eck- } \\
\text { ardt } \\
\text { et al. } \\
{[17]}\end{array}$ & $\begin{array}{l}\text { Syno- } \\
\text { vial } \\
\text { sarco- } \\
\text { ma }\end{array}$ & $\begin{array}{l}\text { Right } \\
\text { fore- } \\
\text { arm }\end{array}$ & $\begin{array}{l}3.5 \mathrm{~Gy} \times \\
8 \text { (28 Gy) } \\
\text { preop, } \\
\text { followed by } \\
2 \mathrm{~Gy} \times 10 \\
(20 \mathrm{~Gy}) \\
\text { boost }\end{array}$ & $\begin{array}{l}\text { Gemcitabine } \\
\left(900 \mathrm{mg} / \mathrm{m}^{2} \text { on }\right. \\
\text { days } 1 \text { and } 8) \\
\text { and docetaxel } \\
\left(100 \mathrm{mg} / \mathrm{m}^{2}\right. \\
\text { on day } 8) \text { for } \\
2 \text { cycles at } 21 \\
\text { and } 28 \text { days, } \\
\text { respectively }\end{array}$ & $\begin{array}{l}5 \\
\text { days }\end{array}$ & $\begin{array}{l}40 \\
\text { days }\end{array}$ & $\begin{array}{l}\text { Swelling of } \\
\text { the right fore- } \\
\text { arm with } \\
\text { progressively } \\
\text { worsening } \\
\text { range of motion, } \\
\text { compartment } \\
\text { syndrome }\end{array}$ & $\begin{array}{l}\text { No (last dose } \\
7 \text { days } \\
\text { before } \\
\text { onset) } \\
\end{array}$ & $\begin{array}{l}\text { Edema of the } \\
\text { flexor compart- } \\
\text { ment muscles, } \\
\text { with layering } \\
\text { fluid along the } \\
\text { superficial fascia } \\
\text { and between the } \\
\text { muscles (MRI) }\end{array}$ & $\begin{array}{l}\text { Dexameth- } \\
\text { asone }\end{array}$ & $\begin{array}{l}\text { Patient re- } \\
\text { quired slow } \\
\text { taper cortico- } \\
\text { steroids for } \\
\text { multiple } \\
\text { months; patient } \\
\text { continues to } \\
\text { have muscle } \\
\text { edema and } \\
\text { myositis on 1- } \\
\text { year follow-up } \\
\text { MRI. }\end{array}$ & $\begin{array}{l}\text { No } \\
\text { t } \\
\end{array}$ \\
\hline $\begin{array}{l}\text { Fakih } \\
{[20]}\end{array}$ & $\begin{array}{l}\text { Pancre- } \\
\text { atic } \\
\text { adeno- } \\
\text { carci- } \\
\text { noma }\end{array}$ & $\begin{array}{l}\text { Pan- } \\
\text { creas }\end{array}$ & $\begin{array}{l}1.8 \mathrm{~Gy} \times \\
28 \\
(50.4 \mathrm{~Gy})\end{array}$ & $\begin{array}{l}\text { Concurrent } \\
\text { fluorouracil } \\
\left(2,000 \mathrm{mg} / \mathrm{m}^{2} / \text { day }\right. \\
\text { for } 5 \text { days a week) } \\
\text { and gemcitabine } \\
\text { ( } 200 \mathrm{mg} / \mathrm{m}^{2} \text { weekly) } \\
\text { followed by adjuvant } \\
\text { gemcitabine }(1,000 \\
\mathrm{mg} / \mathrm{m}^{2} / \text { week for } 3 \\
\text { weeks every } \\
\text { 4-week cycle) }\end{array}$ & $\begin{array}{l}0 \text { days } \\
\text { (concurrent) } \\
\sim 21 \text { days } \\
\text { to initi- } \\
\text { ation of } \\
\text { adjuvant dose } \\
\text { gemcitabine }\end{array}$ & $\begin{array}{l}\sim 18 \\
\text { weeks }\end{array}$ & $\begin{array}{l}\text { Erythematosus } \\
\text { rash overlying a } \\
\text { tender mass in } \\
\text { the epigastrium }\end{array}$ & Yes & $\begin{array}{l}\text { Enlarged left } \\
\text { and right } \\
\text { rectus } \\
\text { abdominus } \\
\text { with areas of } \\
\text { heterogeneity } \\
\text { (CT) }\end{array}$ & $\begin{array}{l}\text { None, other } \\
\text { than } \\
\text { withholding } \\
\text { gemcitabine }\end{array}$ & $\begin{array}{l}\text { Complete } \\
\text { resolution }\end{array}$ & $\begin{array}{l}\text { Yes } \\
\text { (capecita- } \\
\text { bine, } \\
\text { docetaxel, } \\
\text { and } \\
\text { cisplatin) }\end{array}$ \\
\hline $\begin{array}{l}\text { Fo- } \\
\text { garty } \\
\text { et al. } \\
{[21]}\end{array}$ & NSCLC & Lung & $\begin{array}{l}3 \mathrm{~Gy} \times \\
12 \\
(36 \mathrm{~Gy})\end{array}$ & $\begin{array}{l}\text { Gemcitabine } \\
\left(1,000 \mathrm{mg} / \mathrm{m}^{2}\right. \\
\text { on days } 1 \text { and } 8) \\
\text { and carboplatin } \\
\text { (AUC } 5 \text {, day } 1 \text { ) }\end{array}$ & $\begin{array}{l}\sim 3 \\
\text { months }\end{array}$ & $\begin{array}{l}4.5 \\
\text { months }\end{array}$ & $\begin{array}{l}\text { Posterior chest } \\
\text { wall pain with } \\
\text { localized } \\
\text { tenderness, skin } \\
\text { rash, elevated } \\
\text { CK, ESR }\end{array}$ & Yes & $\begin{array}{l}\text { Enhancement } \\
\text { of the chest wall } \\
\text { musculature } \\
\text { consistent with } \\
\text { nonspecific } \\
\text { inflammatory } \\
\text { change (MRI) }\end{array}$ & $\begin{array}{l}\text { NSAIDS, oral } \\
\text { steroids }\end{array}$ & $\begin{array}{l}\text { Symptoms } \\
\text { improved } \\
\text { but persistent } \\
\text { minor skin } \\
\text { changes and } \\
\text { subcutaneous } \\
\text { fibrosis }\end{array}$ & $\begin{array}{l}\text { Not re- } \\
\text { ported }\end{array}$ \\
\hline
\end{tabular}


Ravishankar et al.: Gemcitabine-Induced Radiation Recall Myositis: Case Report and

Review of the Literature

Table 2. Collection of previous case reports of gemcitabine-induced radiation recall myositis (continued)

\begin{tabular}{|c|c|c|c|c|c|c|c|c|c|c|c|c|}
\hline Author & $\begin{array}{l}\text { Cancer } \\
\text { type }\end{array}$ & $\begin{array}{l}\text { Radia- } \\
\text { tion } \\
\text { loca- } \\
\text { tion }\end{array}$ & Dose & $\begin{array}{l}\text { CTx } \\
\text { regimen }\end{array}$ & $\begin{array}{l}\text { Time } \\
\text { between } \\
\text { RT and } \\
\text { gemcita- } \\
\text { bine start }\end{array}$ & $\begin{array}{l}\text { Time be- } \\
\text { tween RT } \\
\text { completion } \\
\text { and recall } \\
\text { symptoms }\end{array}$ & $\begin{array}{l}\text { Clinical } \\
\text { symptoms/ } \\
\text { signs }\end{array}$ & $\begin{array}{l}\text { Undergoing } \\
\text { chemo at } \\
\text { time of } \\
\text { symptom } \\
\text { onset? }\end{array}$ & $\begin{array}{l}\text { Imaging } \\
\text { findings } \\
\text { (and } \\
\text { modality) }\end{array}$ & $\begin{array}{l}\text { Treat- } \\
\text { ment }\end{array}$ & Outcome & $\begin{array}{l}\text { Post- } \\
\text { treatment } \\
\text { CTx? }\end{array}$ \\
\hline $\begin{array}{l}\text { Fried- } \\
\text { lander } \\
\text { et al. } \\
\text { [7] }\end{array}$ & $\begin{array}{l}\text { Pancre- } \\
\text { atic } \\
\text { adeno- } \\
\text { carci- } \\
\text { noma }\end{array}$ & $\begin{array}{l}\text { Pan- } \\
\text { creas } \\
\text { and } \\
\text { re- } \\
\text { gional } \\
\text { lymph } \\
\text { nodes }\end{array}$ & $\begin{array}{l}1.8 \mathrm{~Gy} \\
\times 28 \\
(50.4 \\
\text { Gy) }\end{array}$ & $\begin{array}{l}\text { Gemcitabine } \\
\left(40 \mathrm{mg} / \mathrm{m}^{2}\right. \\
\text { biweekly and } \\
\text { concurrently } \\
\text { with radiation, } \\
\text { followed by } \\
1,000 \mathrm{mg} / \mathrm{m}^{2} \\
\text { weekly for } 3 \\
\text { weeks per month) }\end{array}$ & 39 days & $\begin{array}{l}3 \\
\text { months }\end{array}$ & $\begin{array}{l}\text { Tenderness } \\
\text { of rectus } \\
\text { muscles, } \\
\text { mild rash, } \\
\text { elevated CK }\end{array}$ & Yes & $\begin{array}{l}\text { Increased } \\
\text { signal in the } \\
\text { subcutaneous } \\
\text { tissue of the } \\
\text { anterior } \\
\text { abdominal } \\
\text { wall (MRI) }\end{array}$ & $\begin{array}{l}\text { Cortico- } \\
\text { steroids }\end{array}$ & $\begin{array}{l}\text { Complete } \\
\text { resolution, } \\
\text { no recurrence } \\
\text { after steroid } \\
\text { tapering }\end{array}$ & $\begin{array}{l}\text { Not } \\
\text { reported }\end{array}$ \\
\hline $\begin{array}{l}\text { Ganem } \\
\text { et al. } \\
{[22]}\end{array}$ & $\begin{array}{l}\text { Squa- } \\
\text { mous } \\
\text { cell car- } \\
\text { cinoma } \\
\text { of the } \\
\text { lung }\end{array}$ & Pelvis & $\begin{array}{l}3 \text { Gy } \\
\times 11 \\
(33 \\
\text { Gy) }\end{array}$ & $\begin{array}{l}\text { Gemcitabine } \\
\left(1,000 \mathrm{mg} / \mathrm{m}^{2}\right. \\
\text { on days } 1,8,15) \\
\text { and cisplatin } \\
\left(100 \mathrm{mg} / \mathrm{m}^{2} \text { on }\right. \\
\text { day } 15)\end{array}$ & $\begin{array}{l}1.5 \\
\text { months }\end{array}$ & $\begin{array}{l}5 \\
\text { months }\end{array}$ & $\begin{array}{l}\text { Right } \\
\text { buttock } \\
\text { pain }\end{array}$ & Yes & $\begin{array}{l}\text { Hypersignal } \\
\text { and edema on } \\
\text { gluteal soft } \\
\text { tissue (MRI) }\end{array}$ & $\begin{array}{l}\text { Oral } \\
\text { opiates, } \\
\text { antibiotics, } \\
\text { steroids }\end{array}$ & $\begin{array}{l}\text { Alleviation } \\
\text { over the } \\
\text { course of } 3 \\
\text { months }\end{array}$ & $\begin{array}{l}\text { Not } \\
\text { reported }\end{array}$ \\
\hline $\begin{array}{l}\text { Graf } \\
\text { et al. } \\
{[16]}\end{array}$ & $\begin{array}{l}\text { NSCLC } \\
\text { and anal } \\
\text { cancer, } \\
\text { history } \\
\text { of der- } \\
\text { mato- } \\
\text { myositis }\end{array}$ & Pelvis & $\begin{array}{l}\text { Not } \\
\text { re- } \\
\text { port- } \\
\text { ed }\end{array}$ & $\begin{array}{l}\text { 5-FU and MMC } \\
\text { given with } \\
\text { pelvic RT for } \\
\text { anal cancer, } \\
\text { carboplatin } \\
\text { and } \\
\text { gemcitabine } \\
\text { (dosage not } \\
\text { given) }\end{array}$ & $\begin{array}{l}2 \\
\text { months }\end{array}$ & $\begin{array}{l}4 \\
\text { months }\end{array}$ & $\begin{array}{l}\text { Erythema, } \\
\text { swelling, } \\
\text { warmth, } \\
\text { and } \\
\text { tenderness } \\
\text { of the } \\
\text { buttocks } \\
\text { and groin } \\
\text { area }\end{array}$ & Yes & $\begin{array}{l}\text { High signal in the } \\
\text { bilateral gluteal } \\
\text { maximus, } \\
\text { quadratus femo- } \\
\text { ris, adductor mag- } \\
\text { nus, obturator } \\
\text { externus and right } \\
\text { iliopsoas muscles } \\
\text { (MRI), elevated CK }\end{array}$ & $\begin{array}{l}\text { Prednisone } \\
\text { and opiate } \\
\text { analgesia }\end{array}$ & $\begin{array}{l}\text { Gradual } \\
\text { improvement } \\
\text { with steroids }\end{array}$ & $\begin{array}{l}\text { Not } \\
\text { reported }\end{array}$ \\
\hline $\begin{array}{l}\text { Grover } \\
\text { et al. } \\
\text { [5] }\end{array}$ & $\begin{array}{l}\text { Adeno- } \\
\text { carcino- } \\
\text { ma and } \\
\text { neuro- } \\
\text { endocrine } \\
\text { neoplasm, } \\
\text { unknown } \\
\text { primary }\end{array}$ & $\begin{array}{l}\text { Left } \\
\text { hip } \\
\text { and } \\
\text { left } \\
\text { acro- } \\
\text { mion }\end{array}$ & $\begin{array}{l}3 \text { Gy } \\
\times 10 \\
(30 \\
\text { Gy })\end{array}$ & $\begin{array}{l}\text { Gemcitabine } \\
\left(1,250 \mathrm{mg} / \mathrm{m}^{2}\right) \\
\text { and carboplatin } \\
\text { (AUC 5) }\end{array}$ & $\begin{array}{l}2 \\
\text { weeks }\end{array}$ & $\begin{array}{l}4 \\
\text { weeks }\end{array}$ & $\begin{array}{l}\text { Worsening } \\
\text { pain in left } \\
\text { shoulder } \\
\text { and hip }\end{array}$ & Yes & $\begin{array}{l}\text { Soft tissue } \\
\text { edema of the } \\
\text { muscles } \\
\text { adjacent to the } \\
\text { left acromion } \\
\text { and the left } \\
\text { hip (MRI) }\end{array}$ & Narcotics & $\begin{array}{l}\text { Pain resolved } \\
5 \text { months } \\
\text { after radio- } \\
\text { therapy }\end{array}$ & $\begin{array}{l}\text { Gemcitabine } \\
\text { therapy } \\
\text { continued }\end{array}$ \\
\hline $\begin{array}{l}\text { Horan } \\
\text { et al. } \\
{[23]}\end{array}$ & NSCLC & Lung & $\begin{array}{l}3 \text { Gy } \\
\times 8 \\
(24 \\
\text { Gy) }\end{array}$ & $\begin{array}{l}\text { Gemcitabine } \\
\left(1,000 \mathrm{mg} / \mathrm{m}^{2} \text {, }\right. \\
\text { weekly })\end{array}$ & $\begin{array}{l}2 \\
\text { months }\end{array}$ & $\begin{array}{l}\sim 13 \\
\text { weeks }\end{array}$ & $\begin{array}{l}\text { Pain and } \\
\text { swelling of } \\
\text { the right } \\
\text { pectoralis } \\
\text { major, biopsy } \\
\text { proven mus- } \\
\text { cle necrosis } \\
\end{array}$ & Yes & $\begin{array}{l}\text { Thickening of } \\
\text { right pecto- } \\
\text { ralis major } \\
\text { muscle (CT) }\end{array}$ & Analgesics & $\begin{array}{l}\text { Symptoms } \\
\text { gradually } \\
\text { declined } \\
\text { when gemci- } \\
\text { tabine was } \\
\text { stopped }\end{array}$ & $\begin{array}{l}\text { Gemcitabine } \\
\text { re-challenge, } \\
\text { no further } \\
\text { symptoms }\end{array}$ \\
\hline $\begin{array}{l}\text { Jeter } \\
\text { et al. } \\
{[11]}\end{array}$ & $\begin{array}{l}\text { Pancre- } \\
\text { atic } \\
\text { adeno- } \\
\text { carci- } \\
\text { noma }\end{array}$ & $\begin{array}{l}\text { Pan- } \\
\text { creas }\end{array}$ & $\begin{array}{l}1.8 \mathrm{~Gy} \\
\times 28 \\
(50.4 \\
\text { Gy) }\end{array}$ & $\begin{array}{l}\text { Gemcitabine } \\
\left(1,000 \mathrm{mg} / \mathrm{m}^{2}\right. \\
\text { one dose; } \\
\text { followed by } \\
750 \mathrm{mg} / \mathrm{m}^{2} \\
\text { weekly for } 9 \\
\text { months) }\end{array}$ & $\begin{array}{l}3 \\
\text { weeks }\end{array}$ & $\begin{array}{l}3 \\
\text { months }\end{array}$ & $\begin{array}{l}\text { Abdominal } \\
\text { wall } \\
\text { tenderness } \\
\text { and } \\
\text { erythema }\end{array}$ & Yes & $\begin{array}{l}\text { Subcutaneous } \\
\text { fat stranding } \\
\text { and decreased } \\
\text { density of rec- } \\
\text { tus muscles in } \\
\text { radiation } \\
\text { portal (CT) }\end{array}$ & Ibuprofen & $\begin{array}{l}\text { Symptoms } \\
\text { responsive } \\
\text { to ibuprofen }\end{array}$ & $\begin{array}{l}\text { Gemcitabine } \\
\text { re-challenge, } \\
\text { no further } \\
\text { symptoms }\end{array}$ \\
\hline $\begin{array}{l}\text { Lock } \\
\text { et al. } \\
{[24]}\end{array}$ & $\begin{array}{l}\text { Hepatic } \\
\text { adeno- } \\
\text { carci- } \\
\text { noma }\end{array}$ & Liver & $\begin{array}{l}2.94 \\
\text { Gy } \times \\
15 \\
(44.1 \\
\text { Gy) }\end{array}$ & $\begin{array}{l}\text { Gemcitabine } \\
\left(1,000 \mathrm{mg} / \mathrm{m}^{2}\right. \\
\text { for days } 1 \text { and } \\
8 \text { for a 3-week } \\
\text { cycle) }\end{array}$ & $\begin{array}{l}8 \\
\text { weeks }\end{array}$ & $\begin{array}{l}18 \\
\text { weeks }\end{array}$ & $\begin{array}{l}\text { Abdominal } \\
\text { discomfort } \\
\text { with indura- } \\
\text { tion; over- } \\
\text { lying skin } \\
\text { erythema }\end{array}$ & Yes & $\begin{array}{l}\text { Enhancement } \\
\text { of abdominal } \\
\text { muscles with } \\
\text { thickening } \\
\text { (MRI) }\end{array}$ & $\begin{array}{l}\text { Ibuprofen, } \\
\text { vitamin E, } \\
\text { and } \\
\text { vitamin C }\end{array}$ & $\begin{array}{l}\text { Gradual res- } \\
\text { olution over } \\
\text { the course of } \\
6 \text { weeks }\end{array}$ & $\begin{array}{l}\text { Gemcitabine } \\
\text { was contin- } \\
\text { ued, reduction } \\
\text { of symptoms }\end{array}$ \\
\hline $\begin{array}{l}\text { Miura } \\
\text { et al. } \\
{[25]^{a}}\end{array}$ & NSCLC & $\begin{array}{l}\text { Right } \\
\text { hip }\end{array}$ & $\begin{array}{l}2 \text { Gy } \\
\times 25 \\
(50 \\
\text { Gy) }\end{array}$ & $\begin{array}{l}\text { Concurrent cisplatin } \\
\left(80 \mathrm{mg} / \mathrm{m}^{2} \text { day } 1 \text { ) }\right. \\
\text { and vinorelbine ( } 20 \\
\mathrm{mg} / \mathrm{m}^{2} \text {, days } 1,8 \text {, } \\
\text { and } 15 \text { ) followed by } \\
\text { gemcitabine ( } 800 \\
\mathrm{mg} / \mathrm{m}^{2} \text { biweekly) }\end{array}$ & 1 month & 3 months & $\begin{array}{l}\text { Right thigh } \\
\text { pain }\end{array}$ & N/A & $\begin{array}{l}\text { Edema within } \\
\text { right thigh } \\
\text { muscles (MRI) }\end{array}$ & Analgesics & $\begin{array}{l}\text { Gradual } \\
\text { resolution } \\
\text { of symptoms }\end{array}$ & $\begin{array}{l}\text { Yes } \\
\text { (unknown } \\
\text { regimen) }\end{array}$ \\
\hline
\end{tabular}




\section{Case Reports in Oncology}

Ravishankar et al.: Gemcitabine-Induced Radiation Recall Myositis: Case Report and

Review of the Literature

Table 3. Collection of previous case reports of gemcitabine-induced radiation recall myositis (continued)

\begin{tabular}{|c|c|c|c|c|c|c|c|c|c|c|c|c|}
\hline Author & $\begin{array}{l}\text { Cancer } \\
\text { type }\end{array}$ & $\begin{array}{l}\text { Radia- } \\
\text { tion } \\
\text { loca- } \\
\text { tion }\end{array}$ & Dose & $\begin{array}{l}\text { CTx } \\
\text { regimen }\end{array}$ & $\begin{array}{l}\text { Time } \\
\text { between } \\
\text { RT and } \\
\text { gemcitabine } \\
\text { start }\end{array}$ & $\begin{array}{l}\text { Time be- } \\
\text { tween } \\
\text { RT com- } \\
\text { pletion } \\
\text { and recall } \\
\text { symptoms }\end{array}$ & $\begin{array}{l}\text { Clinical symp- } \\
\text { toms/ } \\
\text { signs }\end{array}$ & $\begin{array}{l}\text { Under- } \\
\text { going } \\
\text { chemo at } \\
\text { time of } \\
\text { symptom } \\
\text { onset? }\end{array}$ & $\begin{array}{l}\text { Imaging } \\
\text { findings } \\
\text { (and } \\
\text { modality) }\end{array}$ & Treatment & Outcome & $\begin{array}{l}\text { Post- } \\
\text { treatment } \\
\text { CTx? }\end{array}$ \\
\hline $\begin{array}{l}\text { Miura } \\
\text { et al. } \\
{[25]^{\mathrm{a}}}\end{array}$ & NSCLC & Lung & $\begin{array}{l}2 \text { Gy } \times \\
30(60 \\
\text { Gy) }\end{array}$ & $\begin{array}{l}\text { Concurrent cis- } \\
\text { platin and ( } 80 \\
\mathrm{mg} / \mathrm{m}^{2} \text { day } 1 \text { ) and } \\
\text { vinorelbine ( } 20 \\
\mathrm{mg} / \mathrm{m}^{2} \text {, days } 1 \\
\text { and } 8 \text { ) followed } \\
\text { by vinorelbine } \\
\left(13 \mathrm{mg} / \mathrm{m}^{2} \text { bi- }\right. \\
\text { weekly) and } \\
\text { gemcitabine ( } 800 \\
\mathrm{mg} / \mathrm{m}^{2} \text { biweekly) }\end{array}$ & 3 months & $\begin{array}{l}5.5 \\
\text { months }\end{array}$ & $\begin{array}{l}\text { Upper chest } \\
\text { muscle pain }\end{array}$ & $\mathrm{N} / \mathrm{A}$ & $\begin{array}{l}\text { Enhancement } \\
\text { of pectoralis } \\
\text { muscles (MRI) }\end{array}$ & NSAIDs & $\begin{array}{l}\text { Improvement } \\
\text { of symptoms }\end{array}$ & $\begin{array}{l}\text { Yes (ge- } \\
\text { fitinib) }\end{array}$ \\
\hline $\begin{array}{l}0^{\prime} \text { Regan } \\
\text { et al. } \\
{[10]}\end{array}$ & $\begin{array}{l}\text { Hodgkin } \\
\text { lymphoma }\end{array}$ & Chest & $\begin{array}{l}1.8 \text { Gy } \times \\
22(39.6 \\
\text { Gy) } \\
\end{array}$ & $\begin{array}{l}4 \text { cycles of } \\
\text { gemcitabine, } \\
\text { vinorelbine, } \\
\text { and liposomal } \\
\text { doxorubicin } \\
\text { (unknown } \\
\text { dosage) } \\
\end{array}$ & 2 months & $\begin{array}{l}5 \\
\text { months }\end{array}$ & $\begin{array}{l}\text { Worsening } \\
\text { bilateral } \\
\text { anterior } \\
\text { chest pain, } \\
\text { pectoralis } \\
\text { muscle necrosis } \\
\text { by biopsy }\end{array}$ & $\begin{array}{l}\text { No (last } \\
\text { dose } 2.5 \\
\text { weeks } \\
\text { before } \\
\text { presen- } \\
\text { tation) }\end{array}$ & $\begin{array}{l}\text { Diffuse bilateral } \\
\text { swelling of the } \\
\text { pectoral muscles } \\
\text { with mild stranding } \\
\text { of the adjacent } \\
\text { subcutaneous fat } \\
\text { (CT) }\end{array}$ & Analgesics & $\begin{array}{l}\text { Complete } \\
\text { resolution }\end{array}$ & $\begin{array}{l}\text { Not } \\
\text { reported }\end{array}$ \\
\hline $\begin{array}{l}\text { Patel } \\
\text { et al. } \\
{[26]}\end{array}$ & $\begin{array}{l}\text { Naso- } \\
\text { pha- } \\
\text { ryngeal } \\
\text { carci- } \\
\text { noma }\end{array}$ & $\begin{array}{l}\text { Head } \\
\text { and } \\
\text { neck }\end{array}$ & $\begin{array}{l}70.2 \mathrm{~Gy} \\
\text { total } \\
\text { (fractions } \\
\text { N/A) }\end{array}$ & $\begin{array}{l}\text { Gemcitabine } \\
\left(1,000 \mathrm{mg} / \mathrm{m}^{2}\right) \\
\text { and oxaliplatin } \\
\left(100 \mathrm{mg} / \mathrm{m}^{2}\right) \\
\text { every } 2 \text { weeks }\end{array}$ & $\begin{array}{l}\mathrm{N} / \mathrm{A}(\mathrm{CTx} \\
\text { started } \\
\text { after RT) }\end{array}$ & $\begin{array}{l}6 \\
\text { months }\end{array}$ & $\begin{array}{l}\text { Bilateral neck } \\
\text { pain and } \\
\text { swelling with } \\
\text { restriction of } \\
\text { neck movement }\end{array}$ & Yes & $\begin{array}{l}\text { Diffuse bilateral } \\
\text { soft-tissue } \\
\text { edema of the } \\
\text { muscles in the } \\
\text { cervical neck } \\
\text { (MRI) }\end{array}$ & $\begin{array}{l}\text { Dexame- } \\
\text { thasone }\end{array}$ & $\begin{array}{l}\text { Symptoms } \\
\text { worsened with } \\
\text { tapering of dexame- } \\
\text { thasone/ low-dose } \\
\text { prednisone started } \\
\text { without recurrence. }\end{array}$ & $\begin{array}{l}\text { Not } \\
\text { reported }\end{array}$ \\
\hline $\begin{array}{l}\text { Pinson } \\
\text { et al. } \\
{[27]^{\mathrm{b}}}\end{array}$ & NSCLC & Lung & $\begin{array}{l}3 \mathrm{~Gy} \times \\
10(30 \\
\text { Gy) }\end{array}$ & $\begin{array}{l}\text { Carboplatin (AUC } \\
5 \text { on day } 1 \text { ) and } \\
\text { gemcitabine } \\
\left(1,000 \mathrm{mg} / \mathrm{m}^{2}\right. \\
\text { on days } 1 \text { and } \\
\text { 8), 3-week cycle }\end{array}$ & 4 weeks & $\begin{array}{l}14 \\
\text { weeks }\end{array}$ & $\begin{array}{l}\text { Skin erythema, } \\
\text { upper chest } \\
\text { muscle pain }\end{array}$ & Yes & $\begin{array}{l}\text { Swelling of the } \\
\text { pectoralis major } \\
\text { and pectoralis } \\
\text { minor (CT) }\end{array}$ & Ibuprofen & $\begin{array}{l}\text { Complete } \\
\text { resolution in } \\
3 \text { weeks }\end{array}$ & $\begin{array}{l}\text { Not } \\
\text { reported }\end{array}$ \\
\hline $\begin{array}{l}\text { Squire } \\
\text { et al. } \\
\text { [14] }\end{array}$ & NSCLC & $\begin{array}{l}\text { Pelvis } \\
\text { (left } \\
\text { sacroiliac } \\
\text { and left } \\
\text { aceta- } \\
\text { bulum) }\end{array}$ & $\begin{array}{l}3 \mathrm{~Gy} \times \\
10(30 \\
\text { Gy) }\end{array}$ & $\begin{array}{l}\text { Gemcitabine } \\
\left(1,000 \mathrm{mg} / \mathrm{m}^{2}\right)\end{array}$ & 1 month & $\begin{array}{l}3 \\
\text { months }\end{array}$ & $\begin{array}{l}\text { Tenderness } \\
\text { and discomfort } \\
\text { to left hip and } \\
\text { buttock, } \\
\text { elevated CK }\end{array}$ & Yes & $\begin{array}{l}\text { Edema in gluteal } \\
\text { muscles (MRI) }\end{array}$ & $\begin{array}{l}\text { Oral } \\
\text { pred- } \\
\text { nisone }\end{array}$ & $\begin{array}{l}\text { Symptoms } \\
\text { worsened with } \\
\text { tapering of } \\
\text { prednisone } \\
\text { and improved with } \\
\text { increasing doses }\end{array}$ & $\begin{array}{l}\text { Gemcitabine } \\
\text { continued } \\
\text { for } 5 \text { more } \\
\text { months, } \\
\text { symptoms } \\
\text { controlled } \\
\text { with } \\
\text { prednisone } \\
\end{array}$ \\
\hline $\begin{array}{l}\text { Welsh } \\
\text { et al. } \\
\text { [28] }\end{array}$ & $\begin{array}{l}\text { Bladder } \\
\text { cancer }\end{array}$ & $\begin{array}{l}\text { Para- } \\
\text { sacral } \\
\text { region }\end{array}$ & $\begin{array}{l}2.5 \mathrm{~Gy} \\
\times 18 \\
(45 \\
\text { Gy) }\end{array}$ & $\begin{array}{l}\text { Gemcitabine } \\
\text { and cisplatin } \\
\text { (unknown } \\
\text { dosage) }\end{array}$ & 4 weeks & 5 months & $\begin{array}{l}\text { Pain in bilateral } \\
\text { superolateral } \\
\text { gluteal regions }\end{array}$ & Yes & $\begin{array}{l}\text { Band-like } \\
\text { pattern of } \\
\text { edema on } \\
\text { gluteal region } \\
\text { (MRI) }\end{array}$ & $\begin{array}{l}\text { NSAIDs, } \\
\text { pred- } \\
\text { nisone }\end{array}$ & $\begin{array}{l}\text { Complete resolution } \\
\text { after } 6 \text { weeks, but } \\
\text { with visible residual } \\
\text { scar and muscular } \\
\text { atrophy }\end{array}$ & $\begin{array}{l}\text { CTx } \\
\text { continued }\end{array}$ \\
\hline $\begin{array}{l}\text { Cur- } \\
\text { rent }\end{array}$ & $\begin{array}{l}\text { Breast } \\
\text { cancer }\end{array}$ & $\begin{array}{l}\text { Left } \\
\text { thigh } \\
\text { (fe- } \\
\text { mur)/ } \\
\text { T12 } \\
\text { ver- } \\
\text { tebra }\end{array}$ & $\begin{array}{l}3 \text { Gy } \times \\
10(30 \\
\text { Gy)/ } \\
24 \text { Gy } \times \\
1(24 \\
\text { Gy) }\end{array}$ & $\begin{array}{l}\text { Gemcitabine } \\
\left(1,000 \mathrm{mg} / \mathrm{m}^{2}\right. \\
\text { for days } 1 \text { and } \\
8 \text { ) and her- } \\
\text { ceptin } \\
\text { (342 } \mathrm{mg} \\
\text { every } 3 \\
\text { weeks) }\end{array}$ & $\begin{array}{l}54 \text { days/ } \\
79 \text { days }\end{array}$ & $\begin{array}{l}3 \\
\text { months/ } \\
5.7 \\
\text { months }\end{array}$ & $\begin{array}{l}\text { Worsening } \\
\text { leg pain and } \\
\text { swelling/ } \\
\text { Chest and } \\
\text { back pain }\end{array}$ & Yes & $\begin{array}{l}\text { Enlargement of } \\
\text { muscles of the } \\
\text { left thigh (US)/ } \\
\text { T12 fracture and } \\
\text { paraspinal fluid } \\
\text { collections (MRI) }\end{array}$ & $\begin{array}{l}\text { Dexame- } \\
\text { thasone/ } \\
\text { T12 } \\
\text { vertebro- } \\
\text { plasty }\end{array}$ & $\begin{array}{l}\text { Progressive resolu- } \\
\text { tion of symptoms } \\
\text { after 4-month } \\
\text { course of dexame- } \\
\text { thasone/ } \\
\text { Reduction of chest } \\
\text { and back pain } \\
\text { following vertebro- } \\
\text { plasty }\end{array}$ & $\begin{array}{l}\text { Yes (her- } \\
\text { ceptin) }\end{array}$ \\
\hline
\end{tabular}

a Paper is written in Japanese. ${ }^{b}$ Paper is written in Dutch. 